\title{
A case of gonococcal mastitis in a male
}

\author{
Neil J Bodsworth, Ross Price, Megan J Nelson
}

\begin{abstract}
A case of a 42 year old homosexual man with mastitis of the left breast due to Neisseria gonorrhoeae is presented on account of its extreme rarity. It was probably acquired as a result of direct oral-nipple contact.
\end{abstract}

(Genitourin Med 1993;69:222-223)

\section{Case report}

A 42 year old homosexual man presented to a private sexually transmissible disease (STD) clinic in central Sydney with a 3 day history of an enlarging tender mass in his left breast. Ten days before presentation he attended a dance party where an open nipple ring (see figure) became caught in a passerby's armlet on the dance floor. The next day he noted a scant clear discharge with crusting of the lateral ring orifice. This resolved following two days of self treatment with a peroxide solution.

Other than a regular sexual partner of 8

Sydney Sexual Health Centre, Sydney Hospital, Sydney. N J Bodsworth

Taylor Square Private Clinic, Darlinghurst, NSW, Australia R Price

M J Nelson

Address correspondence to: Dr Neil Bodsworth, Sydney Sexual Health Centre, Sydney Hospital, PO Box 1614, Sydney, NSW 2001 Australia.

Accepted for publication 28 January 1993

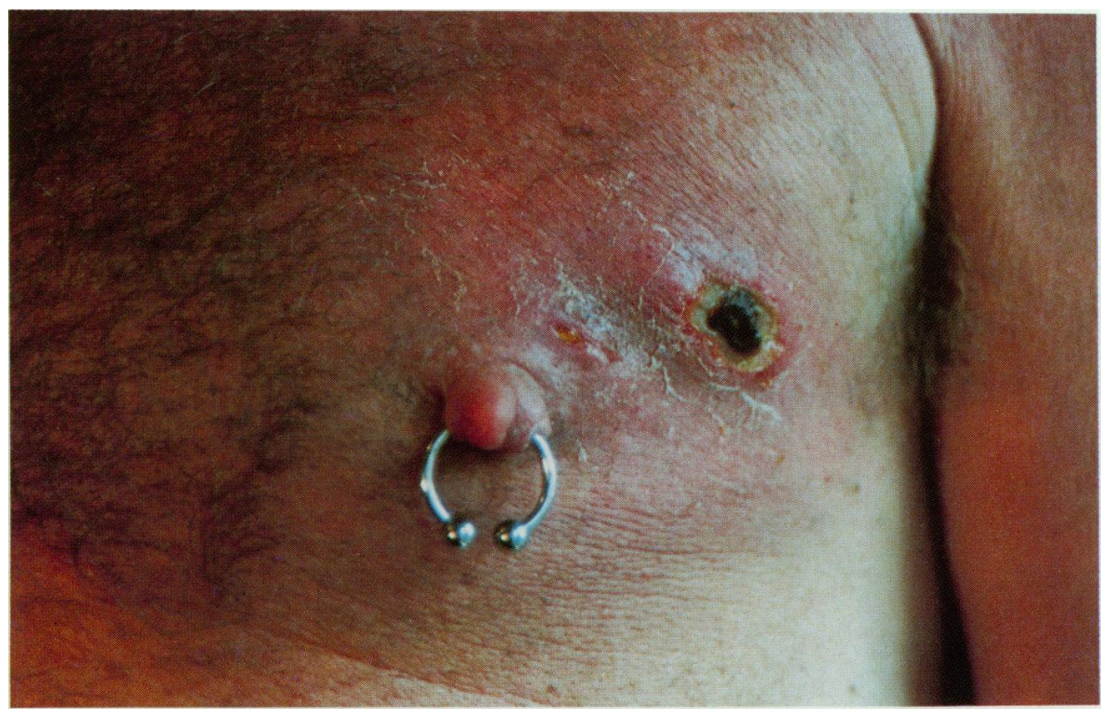

Figure Gonococcal mastitis in a male. ture sites were clean and dry and there were no changes to the skin overlying the breas. The patient was afebrile and there was 100 axillary lymphadenopathy. A diagnosis of non-specific mastitis was made, no tests wefe performed and he was commenced \& flucloxacillin $500 \mathrm{mg}$ orally q.i.d.

At review six days later, two abscesses had formed, one at the site of the original mass and another further towards the axilla. He remained afebrile and was generally well. Fisse $\mathrm{ml}$ of pus was drained from the media abscess which produced a heavy growth of Neisseria gonorrhoeae (confirmed by carbiohydrate degradation, method of Tapsall and Cheng). ${ }^{1}$ The isolate was beta lactamase negative but relatively resistant to penicillin (mean inhibitory concentration [MIE] $1.0 \mathrm{mg} / \mathrm{l}$.) The serotype was $\mathrm{lb} 4$ and the were no particular amino acid requirements for growth (prototrophic auxotype). CA magnoplasm poultice was applied to the less organised second abscess.

Ciprofloxacin $500 \mathrm{mg}$ bid was added to treatment when the culture result becanne available two days later. Blood, urethral and throat cultures taken at this time were all ne $\overrightarrow{\text { g }}-$ ative for $N$. gonorrhoeae. Full blood examina tion revealed a mild leucocytosis $(12 \cdot 8$ $10^{9} / \mathrm{I} ; 75 \%$ neutrophils). HIV antibody was negative in 1986 and he declined retesting because of perceived low risk. Throat and urethral cultures of the regular partner wexe negative for $N$. gonorrhoeae. Unfortunately the American tourist could not be contacted.

Four days after the application of the pou tice the second abscess burst resulting in the appearance of the figure. Both woundis resolved completely over the following five days.

\section{Discussion}

We believe this to be the first report of masttis due to infection with Neisseria gonorrhoigge in either man or woman.

Mastitis in a man has previously be $\overrightarrow{\mathbb{E}}$ reported as a complication of mumps ${ }^{2}$ but $\frac{1}{6}$ are unaware of any cases that result from direct inoculation. There are occasional reports of mastitis in neonates of both sexes, presumably following ductal hypertrophy sefondary to maternal oestrogen. In aduilt women mastitis is usually seen in nursing mothers with $S$. aureus the commonest pathogen isolated. $E$. coli, enterococci and streptococci are less common causes and reports have appeared implicating histoplas- 
ma, ${ }^{3}$ M. tuberculosis, ${ }^{4}$ M. fortuitum, ${ }^{5}$ Salmonella (typhi $i^{6}$ and non-typhi $i^{7}$ ) and anaerobes. ${ }^{8}$

Gonococcal abscesses can occur as a result of direct inoculation, local spread or haematogenous metastasis from the site of primary infection. They occurred more frequently in the pre-antibiotic era. In 1923 Thompson cited reports of abscesses in compound fractures, the mastoid process and various muscles. ${ }^{9} \mathrm{He}$ also cited cases of the gonococcus causing: pneumonia, phlebitis, endocarditis, myocarditis, pericarditis, peritonitis, cystitis, pyelonephritis, otitis, meningitis, and most commonly arthritis and cutaneous abscesses.

The present case probably results from direct transmission of $N$. gonorrhoeae from the mouth of the American tourist rather than metastasis from another site of the index case. $N$. gonorrhoeae was not isolated from the urethra, pharynx or blood of the index case nor the urethra or pharynx of his long term partner. However, flucloxacillin had been administered for 9 days before these swabs were obtained. This therapy may have eradicated any pharyngeal or urethral infection despite the relative insensitivity of the isolate to penicillin $(\mathrm{MIC}=1.0 \mathrm{mg} / \mathrm{l})$ and the relative inactivity of flucloxacillin (when compared with penicillin) against $N$. gonorrhoeae. Direct transmission to the parotid gland (a similar exocrine organ) has been reported following fellatio of a man with demonstrated urethral gonorrhoea. ${ }^{10}$

The serovar (Ib4), auxotype (prototrophic), and relative insensitivity to pencillin (MIC $=1.0 \mathrm{mg} / \mathrm{l}$ ) of the current isolate also suggest direct inoculation of the mammary ducts rather than blood-borne dissemination. In a recent report from the Australian Gonococcal Surveillance Programme (AGSP), ${ }^{11}$ disseminated gonococcal infection (DGI) was strongly associated with strains of the Ia serogroup (21 of 22 isolates) and the proline requiring auxotype (17 of 22 isolates). This contrasts with the US experience where most DGI isolates are of the AHU auxotype ${ }^{12}$ which is only very rarely isolated from mucosal sites in Australia. ${ }^{11}$ Moreover, DGI is usually associated with strains of $N$. gonorrhoeae that possess less intrinsic (chromosomal) resistance to penicillin. ${ }^{13}$ All 16 betalactamase negative DGI isolates in the AGSP series had penicillin MICs of less than $0.5 \mathrm{mg} / \mathrm{l}$.

We thank Ms Edna Phillips of the Department of Microbiology, Prince of Wales Hospital for auxotyping the isolate.

1 Tapsall J, Cheng JK. Rapid identification of pathogenic species of Neisseria by carbohydrate degradatio methods: importance of glucose in media used for preparation of inocula. $B \mathcal{f}$ Venereal Dis. 1981;57:249-52.

2 Happel JS. Mastitis in the male-a rare complication of mumps. BMF 1965;5469:1041.

3 Osbome BM. Granulomatous mastitis caused by histoplasma and mimicking inflammatory breast carcinoma. Hum Pathol. 1989;20:47-52.

4 Tan KK, Tan TH. Tuberculosis of the breast. Singapore Med F. 1988;29:271-5.

5 Juang YC, Wang LS, Chen CH, Lin CY. Mycobacterium fortuitum mastitis following augmentation mammaplasty: report of a case. Taiwan-i-Hsueh-Hui-Tsa-Chin 1989;88:278-81.

6 Campbell FC, Eriksson BL, Angorn IB. Localized granulomatous mastitis - an unusual presentation of typhod: case report. $S$ Afr Med $\mathcal{~}$. 1980;57:793-5.

7 Stamm AM. Salmonella bredeney mastitis during pregnancy. Obstet Gynecol. 1982;59(6 suppl):29s-30s.

8 Ingham HR, Freeman R, Wilson RG. Anaerobic breast abscess [letter]. Lancet. 1979;i:164-5.

9 Thompson D. Gonorrhea Oxford Medical Press, London 1923 .

10 Diefebach WCL. Gonorrheal parotitis. Oral Surgery, Oral Medicine and Oral Pathology. 1953;6:974.

11 Tapsall JW, Phillips EA, Shultz Way B, Withnall K, on behalf of The Australian Gonococcal Surveillance Programme. Strain characteristics and antibiotic sucep tibility of isolates of Neisseria gonorrhoeae causing disseminated gonococcal infection in Australia. Int. F AIDS STD. 1992;3:273-7.

12 Knapp JS, Holmes KK. Disseminated gonococcal infections caused by Neisseria gonorrhoeae with unique nutritional requirements. $₹$ Infec Dis $1975 ; 132: 204-8$.

13 Wiesner PJ, Handsfield $\mathrm{HH}$, Holmes KK. Low antibiotic resistance of gonococci causing disseminated infection. N Engl ₹ Med. 1973; 288:1221-2. 